\title{
Schrödinger link between nonequilibrium thermodynamics and Fisher information
}

\author{
B. R. Frieden, ${ }^{1}$ A. Plastino, ${ }^{1,2,3}$ A. R. Plastino, ${ }^{2,3}$ and B. H. Soffer ${ }^{1, *}$ \\ ${ }^{1}$ University of Arizona, Tucson, Arizona 85721 \\ ${ }^{2}$ Universidad Nacional de La Plata, C.C. 727, 1900 La Plata, Argentina \\ ${ }^{3}$ Argentine National Research Center (CONICET), Buenos Aires, Argentina
}

(Received 29 April 2002; revised manuscript received 8 July 2002; published 22 October 2002)

\begin{abstract}
It is known that equilibrium thermodynamics can be deduced from a constrained Fisher information extemizing process. We show here that, more generally, both nonequilibrium and equilibrium thermodynamics can be obtained from such a Fisher treatment. Equilibrium thermodynamics corresponds to the ground-state solution, and nonequilibrium thermodynamics corresponds to excited-state solutions, of a Schrödinger wave equation (SWE). That equation appears as an output of the constrained variational process that extremizes Fisher information. Both equilibrium and nonequilibrium situations can thereby be tackled by one formalism that clearly exhibits the fact that thermodynamics and quantum mechanics can both be expressed in terms of a formal SWE, out of a common informational basis. As an application, we discuss viscosity in dilute gases.
\end{abstract}

DOI: $10.1103 /$ PhysRevE.66.046128

PACS number(s): 05.70.Ln, 05.90.+m, 03.65.-w

\section{INTRODUCTION}

The information content of a normalized probability distribution $P(i) i=1, \ldots, N$, where the index $i$ runs over the states of the system one is trying to study, is given by Shannon's information measure (IM) [1]

$$
S=-\sum_{i=1}^{N} P(i) \ln [P(i)]
$$

The choice of the logarithmic base fixes the information units. If the basis is 2, then $S$ is measured in bits. If one chooses Boltzmann's constant as the informational unit and identifies Shannon's IM with the thermodynamic entropy, then the whole of statistical mechanics can be elegantly reformulated by extremization of Shannon's $S$, subject to the constraints imposed by the a priori information one may possess concerning the system of interest [1].

Now, the phenomenal success of thermodynamics and statistical physics crucially depends upon certain necessary mathematical relationships involving energy and entropy (Legendre transform structure). In the equilibrium situation these relationships are also valid if one replaces $S$ by Fisher's information measure $I$ (FIM) [2]. Using this measure [3], the entire Legendre-transform structure of thermodynamics can be reexpressed (i.e., I replaces the Boltzmann-Shannon $S$ ). In general, this abstract Legendre structure constitutes an essential ingredient that allows one to build up a statistical mechanics. Fisher information $I$ allows then for such a construction. Also, a desired concavity property, obeyed by $I$, further demonstrates its utility as a statistical mechanics generator.

The interested reader might want to consult works by Frieden, Soffer, Nikolov, Plastino, Silver, Hughes, Helstrom, Holevo, Reginatto, Hall, Nettleton, Villani, Casas, and others, that have shed much light upon the manifold physical applications of Fisher's information measure [4-26]. It is

\footnotetext{
*Present address: 665 Bienveneda Avenue, Pacific Palisades, CA 90272.
}

interesting to note that the present work also covers the subject of the classical nonequilibrium description of simple fluids, recently dealt with in a quite interesting fashion from a different angle by Nettleton in [22].

Here we will show that the variational treatment of Fisher information also accounts for nonequilibrium situations. See also [26] in this regard. We will connect Fisher information $I$ with nonequilibrium thermodynamics via the Schrödinger equation (SWE). Such a connection is of interest because it clearly shows that equilibrium and nonequilibrium states have a common informational origin that is expressed by the SWE. The same SWE also allows for quantum scenarios, or even mixed quantum and thermodynamic scenarios.

The paper is organized as follows. For the benefit of the reader we review (i) our Fisher variational treatment of [2] in Sec. II, and (ii) the Rumer and Ryvkin treatment of Boltzmann's transport equation [27] in Sec. III. Our present formalism is developed in Sec. IV. Boltzmann's equation in the so-called relaxation approximation is the subject of Sec. V, while Sec. VI is devoted to the application of our present formalism to viscosity in dilute gases. Finally, some conclusions are drawn in Sec. VII.

\section{FISHER'S INFORMATION MEASURE FOR TRANSLATION FAMILIES: A VARIATIONAL TREATMENT}

Consider a system that is specified by a physical parameter $\theta$ at a given time $t$. Let $g(x, \theta \mid t)$ describe the probability density function (PDF) for this parameter at that time. Of course, by normalization,

$$
\int d x g(x, \theta \mid t)=1
$$

The Fisher information measure (FIM) $I$ is of the form [28]

$$
I=\int d x g\left[\frac{\partial g / d \theta}{g}\right]^{2}, \quad g=g(x, \theta \mid t) .
$$


The special case of translation families is of use. These are monoparametric families of distributions of the form

$$
g(x, \theta \mid t)=p(u \mid t), \quad u \equiv x-\theta,
$$

which are known up to the shift parameter $\theta$. Following Mach's principle, all members of the family possess identical shape $p(u \mid t)$ (there are no absolute origins). Here FIM takes the appearance $[21,26]$

$$
I=\int d x \frac{(\partial p / \partial x)^{2}}{p}, \quad p=p(x \mid t) .
$$

Our present considerations assume one is dealing with coordinates $x$ that belong to $\mathcal{R}$. Let us focus attention upon the positive-definite, normalized PDF $p(x \mid t)$, evaluated at the time $t$. It of course obeys the normalization

$$
\int d x p(x \mid t)=1
$$

Let the mean values

$$
\theta_{k} \equiv\left\langle A_{k}\right\rangle \text { of } M \text { functions } A_{k}(x), \quad k=1, \ldots, M
$$

be measured at the time $t$. By definition,

$$
\left\langle A_{k}\right\rangle_{t}=\int d x A_{k}(x) p(x \mid t), \quad k=1, \ldots, M .
$$

These mean values will play the role of thermodynamic variables, as explained in [2].

It is of importance to note that the prior knowledge (8) represents information at the fixed time $t$. The problem we attack is to find the PDF $p$ that extremizes $I$ subject to prior conditions (6) and (7). Our Fisher-based extremization problem takes the form

$$
\delta_{p}\left\{I(p)-\alpha\langle 1\rangle-\sum_{k}^{M} \lambda_{k}\left\langle A_{k}\right\rangle_{t}\right\}=0, \quad p \equiv p(x \mid t),
$$

at the given time $t$. Equation (9) is equivalent to

$$
\delta_{p}\left\{\int d x\left(\frac{(\partial p / \partial x)^{2}}{p}-\alpha f-\sum_{k}^{M} \lambda_{k} A_{k} p\right)\right\}=0,
$$

where we have introduced the $(M+1)$ Lagrange multipliers $\left(\alpha, \lambda_{1} \cdots \lambda_{M}\right)$, where each Lagrange multiplier $\lambda_{k} \equiv \lambda_{k}(t)$. Variation leads now to

$$
\begin{aligned}
& \int d x \delta p\left\{(p)^{-2}\left(\frac{\partial p}{\partial x}\right)^{2}+\frac{\partial}{\partial x}\left[(2 / p) \frac{\partial p}{\partial x}\right]+\alpha+\sum_{k}^{M} \lambda_{k} A_{k}\right\} \\
& =0
\end{aligned}
$$

and, on account of the arbitrariness of $\delta p$,

$$
\left\{(p)^{-2}\left(\frac{\partial p}{\partial x}\right)^{2}+\frac{\partial}{\partial x}\left[(2 / p) \frac{\partial p}{\partial x}\right]+\alpha+\sum_{k}^{M} \lambda_{k} A_{k}\right\}=0 .
$$

It is clear that the normalization condition on $p$ makes $\alpha$ a function of the $\lambda_{i}$ 's. Let then $p_{I}(x,\{\lambda\})$ be a solution of Eq. (12), where obviously $\{\lambda\}$ is an $M$-dimensioned Lagrange multipliers vector. The extreme Fisher information is now a function of time,

$$
I=\int d x \frac{(\partial p / \partial x)^{2}}{p} \equiv I(t)
$$

since $p=p(x \mid t)$. Since $p$ extremized $I$, we write

$$
p \equiv p_{I}, \quad p_{I} \equiv p_{I}(x \mid t) .
$$

Let us now find the general solution of Eq. (12). For the sake of simplicity, let us define

$$
G(x, t)=\alpha+\sum_{k}^{M} \lambda_{k}(t) A_{k}(x),
$$

and recast Eq. (12) as

$$
\left[\frac{\partial \ln p_{I}}{\partial x}\right]^{2}+2 \frac{\partial^{2} \ln p_{I}}{\partial x^{2}}+G(x)=0 .
$$

We introduce now the identification [13] $p_{I}=(\psi)^{2}$, recalling that $\psi(x)$ can always be assumed real for one-dimensional problems [2]. Introduce now the new functions

$$
v=\frac{\partial \ln \psi}{\partial x}, \quad \psi \equiv \psi(x, t), \quad v \equiv v(x, t) .
$$

Then Eq. (15) simplifies to

$$
v^{\prime}=-\left\{\frac{G}{4}+v^{2}\right\},
$$

where the prime stands for the derivative with respect to $x$. The above equation is a Riccati equation [29]. Introduction further of [29]

$$
u=\exp \left\{\int^{x} d x[v]\right\}, \quad u=u(x, t),
$$

i.e.,

$$
u=\exp \left\{\int^{x} d x \frac{d \ln \psi}{d x}\right\}=\psi,
$$

places Eq. (15) in the form of a Schrödinger wave equation (SWE) [29]

$$
-(1 / 2) \psi^{\prime \prime}-(1 / 8) \sum_{k}^{M} \lambda_{k}(t) A_{k} \psi=\alpha \psi / 8,
$$

where the Lagrange multiplier $\alpha / 8$ plays the role of an energy eigenvalue, and the sum of the $\lambda_{k} A_{k}(x)$ is an effective potential function

$$
U=(1 / 8) \sum_{k}^{M} \lambda_{k}(t) A_{k}, \quad U=U(x, t) .
$$


Note that no specific potential has been assumed, as is appropriate for thermodynamics. Also, we remark that $U$ is a time-dependent potential function and will permit nonequilibrium solutions. The specific $A_{k}(x)$ to be used here depend upon the nature of the physical application at hand [cf. Eq. (8)]. This application could be of either a classical or a quantum nature.

Also notice that Eq. (20) represents a boundary value problem, generally with multiple solutions, in contrast with the unique solution one obtains when employing JaynesShannon's entropy in place of FIM [1]. As discussed in some detail in [2] and [26], the solution leading to the lowest $I$ value is the equilibrium one. That was the only solution discussed there. Here we wish to generalize the concomitant discussion and ask the following: can we choose other solutions?

\section{RUMER AND RYVKIN'S APPROACH TO NONEQUILIBRIUM THERMODYNAMICS}

In Ref. [27], Rumer and Ryvkin (RR) use the conventional Boltzmann transport equation to build up nonequilibrium solutions. They take the following approach.

(i) Consider a nonequilibrium state of a gas after the lapse of a time $t$ large compared to the time of initial randomization. The time $t$ is regarded as fixed.

(ii) The time $t$ is, also, small compared to the macroscopic relaxation time $T^{*}$ for attaining the Maxwell-Boltzmann law $f_{0}$ on velocities.

(iii) At each point of the vessel containing the gas, a state arises which is close to the local equilibrium state in which $f_{0}$ is the Maxwell-Boltzmann law on velocities.

(iv) This allows one to expand the nonequilibrium distribution $f(x \mid t)$ as

$$
f(x, t) / f_{0}=1+\epsilon \chi(x, t)
$$

where $\epsilon$ is small and the function $\chi$ is to be the object of our endeavors.

(v) The unknown function $\chi(x, t)$ may itself be expanded as a series of (orthogonal) Hermite-Gaussian polynomials $H_{i}(x)$ with coefficients $a_{i}(t)$ at the fixed time $t$,

$$
\chi(x, t)=\sum a_{i}(t) H_{i}(x) .
$$

It is important to remark that Hermite-Gaussian polynomials are orthogonal with respect to a Gaussian kernel, i.e., the equilibrium distribution. No other set of functions is orthogonal (and complete) with respect to a Gaussian kernel function.

(vi) Because of orthogonality, the unknown coefficients $a_{i}(t)$ relate linearly to appropriate (unknown) moments of $f$ over velocity space ( $x$ space).

(vii) Substituting the expansion for $f$ into the transport equation and integrating over all velocities yields now a set of first-order differential equations in the moments (which are generally a function of the fixed time value $t$ ). (viii) These are now solvable subject to known initial conditions, like our expectation values. The moments now become known (including any time dependence).

(ix) As a consequence, the coefficients $a_{i}(t)$ of Eq. (23) are also known, which gives $f$.

What does the $f$ as determined above represent? According to Ref. [27], the solution of the above system of equations would be equivalent to the exact solution of Boltzmann's equation (if enough a priori information were available).

We emphasize that RR do not use an SWE in their approach.

\section{CONNECTING THE SWE EXCITED SOLUTIONS TO NONEQUILIBRIUM THERMODYNAMICS}

Returning to our analysis, we ask the following: can the excited SWE solutions to Eq. (20) represent nonequilibrium states of thermodynamics $[11,26]$ ? An interesting discussion of this point is provided in [22]. Here we try to answer this question in a different fashion by considering, again, the case in which $x$ is a velocity and one seeks the nonequilibrium probability $p(x \mid t)$.

Let excited solutions $\psi_{n}(x, t)$ to the SWE Eq. (20) be identified by a subindex value $n>0$. These amplitude functions are superpositions of Hermite-Gaussian polynomials of the form

$$
\psi_{n}(x, t)=\sum_{i} b_{i n}(t) H_{i}(x), \quad n=1,2, \ldots .
$$

The total number of coefficients $b_{n i}(t)$ depends on how far from equilibrium we are. At equilibrium there is only one such coefficient.

We will show that the squares of these amplitudes agree, under certain conditions (see below), with the known solutions of the Boltzmann transport equation [11,21,23]. Our coefficients $b_{i n}(t)$ are computed at the fixed time $t$ at which our input data $\left\langle A_{k}\right\rangle_{t}$ are collected. While the ground-state solution of Eq. (20) gives the equilibrium states of thermodynamics [2], the excited solutions of Eq. (20) will be shown to give nonequilibrium states. For this to happen, our functions $\psi_{n}(x, t)$ will have to be connected to the RR $f(x, t)$ of Eq. (23) via the squaring operation $\psi_{n}^{2}(x, t)$.

Notice that the square of an expansion in HermiteGaussian polynomials is likewise a superposition of Hermite-Gaussian polynomials, with coefficients $c_{i n}(t)$,

$$
\psi_{n}^{2}(x, t)=\sum_{i} c_{i n}(t) H_{i}(x), \quad n=1,2, \ldots
$$

We argue now to the effect that, for fixed $n$, the RR coefficients $a_{i}(t)$ and our $c_{i n}(t)$ are equal.

First of all, the RR coefficients are certainly computed, like ours, at a fixed time $t$. That is, their momenta are evaluated at that time. Likewise ours [the $\left\langle A_{k}\right\rangle$ of Eq. (8)] can be regarded as velocity momenta at that time as well.

The difference between the RR coefficients and ours is one of physical origin, as follows. RR solve for the velocity moments at the fixed time $t$. These $M_{\mathrm{RR}}$ moments are computed using the RR $a_{i}$ of Eq. (23). We, instead, collect as experimental inputs these velocity moments (at the fixed 
time $t$ ). Thus, if the $M_{\mathrm{RR}}$ moments coincide with our experimental inputs, necessarily the $a_{i}(t)$ and the $c_{i n}(t)$ have to coincide well. Let us repeat: the RR moments at the time $t$ are physically correct by construction, since they actually solve for them via use of the Boltzmann transport equation. The premise of our constrained Fisher information approach is that its input constraints (here our velocity moments $\left\langle A_{k}\right\rangle_{t}$ ) are correct, since they come from experiment. (They calculate, we measure.)

If there is no agreement between the RR moments and our experimental inputs, two possibilities come to mind: (a) we are measuring inputs showing strong quantum effects, while the RR treatment cannot handle such a case (being classical), or (b) the number $M$ of available experimental data we use as inputs does not equal the number $M_{\mathrm{RR}}$ of RR computed moments. This possible disagreement is, however, of a logistic rather than fundamental nature.

The required number of expansion coefficients $b_{i}$ in Eq. (24) is of interest. At equilibrium only one is needed $\left(b_{0}\right)$, as that situation is described by a grand-canonical distribution function that is Gaussian. Next, if the system is sufficiently close to equilibrium, then very few are needed. Hence, nearequilbrium cases should pose little numerical difficulty.

Summing up, the approach given in this paper will give exactly the same solutions at the fixed (but arbitrary) time $t$ as does the RR approach. Therefore, for fixed $n$, our $c_{i n}(t)$ 's coincide with the RR $a_{i}(t)$ 's and our $p(x \mid t)$ coincide with the $\operatorname{RR} f(x, t)$. This holds at each time $t$ [cf. Eq. (8)]. For any other time value, $t^{\prime}$, say, we would have to input new $\left\langle A_{k}\right\rangle$ values appropriate for that time. RR, instead, get coefficients $a_{i}(t)$ valid for continuous time $t$, since they are using Boltzmann's transport equation, which is a continuous one. Our approach, by contrast, yields solutions valid at a discrete point of time $t$. This distinction, "discrete versus continuous," does not compromise the validity of the FisherSchrödinger, nonequilibrium thermodynamics bridge that we have built up here. In order to illustrate our formalism with a relevant application, we consider next a special instance that one often encounters in dealing with Boltzmann's equation.

\section{BOLTZMANN EQUATION IN THE RELAXATION APPROXIMATION}

With a view on developing a simple application of our formalism, in considering the celebrated transport equation of Boltzmann's we will focus attention upon a gas in which the effect of molecular collisions is always to restore a local equilibrium situation described by the Maxwell-Boltzmann PDF $f_{0}(\mathbf{r}, \mathbf{v})$ [30]. In other words, we assume that if the molecular distribution is disturbed from the local equilibrium so that the actual PDF $f$ is different from $f_{0}$, then the effect of collisions is simply to restore $f$ to the local equilibrium value $f_{0}$ exponentially with a relaxation time $\tau$ of the order of the average time between molecular collisions.

In symbols, for fixed $\mathbf{r}, \mathbf{v}, f$ changes as a result of collisions according to

$$
f(t)=f_{0}+\left[f-f_{0}\right] \exp -[t / \tau]
$$

In these conditions, the ensuing Boltzmann equation becomes [30]

$$
\frac{\partial f}{\partial t}+\sum_{i=1}^{3}\left[v_{i} \frac{\partial f}{\partial x_{i}}+\dot{v}_{i} \frac{\partial f}{\partial v_{i}}\right]=-\frac{f-f_{0}}{\tau},
$$

a linear differential equation for $f$.

We consider now a situation slightly removed from equilibrium: $f=f_{0}+f_{1}$ with $f_{1} \ll f_{0}$, so that Eq. (27) turns into

$$
\frac{\partial f}{\partial t}+\sum_{i=1}^{3}\left[v_{i} \frac{\partial f}{\partial x_{i}}+\dot{v}_{i} \frac{\partial f}{\partial v_{i}}\right]=-f_{1} / \tau
$$

The left-hand side of Eq. (28) is small, since the righthand side is, by definition, small. As a consequence, we can evaluate it by neglecting terms in $f_{1}$ and write

$$
\frac{\partial f_{0}}{\partial t}+\sum_{i=1}^{3}\left[v_{i} \frac{\partial f_{0}}{\partial x_{i}}+\dot{v}_{i} \frac{\partial f_{0}}{\partial v_{i}}\right]=-f_{1} / \tau .
$$

Since $f_{0}$ is the Maxwell-Boltzmann PDF, independent of time $\left[\left(\partial f_{0} / \partial t\right)=0\right]$, we finally get the so-called Boltzmann equation in the relaxation approximation [30],

$$
\sum_{i=1}^{3}\left[v_{i} \frac{\partial f_{0}}{\partial x_{i}}+\dot{v}_{i} \frac{\partial f_{0}}{\partial v_{i}}\right]=-f_{1} / \tau .
$$

\section{APPLICATION: VISCOSITY}

As a concrete example of our abstract formalism we will apply it here to the nonequilibrium problem posed by the phenomenon of viscosity in dilute gases. We briefly discuss the corresponding phenomenology in Sec. VI A while in Sec. VIB, we find the distribution law [Eq. (54), see below] predicted by the Boltzmann transport theory. Because we have been using the relatively little-known Rumer-Ryvkin approach [27], we also show, in Sec. VIC, that the RR answer (66) for the distribution function agrees with that of the Boltzmann approach. Finally, in Sec. VID we show that the SWE approach gives the same answer as well, i.e., Eq. (66).

\section{A. Generalities}

Imagine, in a gas, some plane with its normal pointing along the $z$ direction. The fluid below this plane exerts a mean force per unit area (stress) $\mathbf{P}_{z}$ on the fluid above the plane. Conversely, the gas above the plane exerts a stress $-\mathbf{P}_{z}$ on the fluid below the plane. The $z$ component of $\mathbf{P}_{z}$ measures the mean pressure $\langle p\rangle$ in the fluid, i.e., $P_{z z}=\langle p\rangle$. When the fluid is in equilibrium (at rest or moving with uniform velocity throughout), then $P_{z z}=0$ [30]. Consider a nonequilibrium situation in which the gas does not move with uniform velocity throughout. In particular, imagine that the fluid has a constant (in time) mean velocity $u_{x}$ in the $x$ direction such that $u_{x}=u_{x}(z)$. For specific examples, see, for instance, [30]. Now any layer of fluid below a plane $z$ $=$ const will exert a tangential stress $P_{z x}$ on the fluid above it. If $\partial u_{z} / \partial z$ is small, one has [30] 


$$
P_{z x}=-\eta \frac{\partial u_{z}}{\partial z}
$$

where $\eta$ is called the viscosity coefficient. The phenomenon was first investigated by Maxwell, who showed that, for a dilute gas of particles of mass $m$ moving with mean velocity $\langle v\rangle$,

$$
\eta \propto n\langle v\rangle m l,
$$

where $n$ is the number of molecules per unit volume and $l$ is the mean free path [30].

Now consider any quantity $\chi(\mathbf{r}, t)$ whose mean value is

$$
\langle\chi(\mathbf{r}, t)\rangle=\frac{1}{n(\mathbf{r}, t)} \int d^{3} v f(\mathbf{r}, \mathbf{v}, t) \chi(\mathbf{r}, t),
$$

with $n(\mathbf{r}, t)$ the mean number of particles, irrespective of velocity, which at time $t$ are located between $\mathbf{r}$ and $\mathbf{r}+d \mathbf{r}$. If $\chi(\mathbf{r}, t) \equiv \mathbf{v}(\mathbf{r}, t)$, the above relation yields the mean velocity $\mathbf{u}(\mathbf{r}, t)$ of a molecule located near $\mathbf{r}$ at time $t . \quad \mathbf{u}(\mathbf{r}, t)$ describes the mean velocity of a flow of gas at a given point, i.e., the (macroscopic) hydrodynamical velocity. The peculiar velocity $\mathbf{U}$ of a molecule is defined in the fashion [30]

$$
\mathbf{U}=\mathbf{v}-\mathbf{u},
$$

so that

$$
\langle\mathbf{U}\rangle=0
$$

If one is interested in transport properties, the fluxes of various quantities become the focus of attention. Consider the net amount of the quantity $\chi$ transported above, (i) per unit time and (ii) per unit area of an element of area oriented along $\hat{\mathbf{n}}$, by molecules with velocity $\mathbf{U}$ due to their random movement back and forth across this element of area. The $\chi$-associated flux $\mathcal{F}_{n}$ generated in this way is

$\mathcal{F}_{n}(\mathbf{r}, t)=\int d^{3} v f(\mathbf{r}, \mathbf{v}, t)[\hat{\mathbf{n}} \cdot \mathbf{U}] \chi(\mathbf{r}, t)=n\langle[\hat{\mathbf{n}} \cdot \mathbf{U}] \chi\rangle$.

For the present discussion we have $\chi=m v_{x}$ and $\hat{\mathbf{n}} \cdot \mathbf{U}$ $=n U_{z}$. The ensuing flux gives then, precisely, $P_{z x}$ [31]. Since $u_{x}$ does not depend upon the velocity,

$$
P_{z x}=n m\left\langle U_{z} v_{x}\right\rangle=n m\left\langle U_{z}\left[u_{x}+U_{x}\right]\right\rangle=n m\left\langle U_{z} U_{x}\right\rangle .
$$

A simple phenomenological line of reasoning that utilizes the so-called path integral approximation yields then [30]

$$
P_{z x}=-\frac{n \tau \partial u_{x}}{\beta \partial z}, \quad \eta=\frac{n \tau}{\beta},
$$

where $\tau$ is the average time between molecular collisions (relaxation time) and $\beta=1 / k T$.

\section{B. Dealing with viscosity in the Boltzmann relaxation approximation}

We shall first discuss how to tackle the viscosity problem along conventional, Boltzmann equation lines. To such an end assume that the effect of collisions is just to produce a local equilibrium distribution relative to the gas moving with a mean velocity $u_{x}$ at the location of each collision. The relevant equilibrium Maxwell-Boltzmann PDF is

$$
\begin{gathered}
f_{0}(\mathbf{r}, \mathbf{v}, t)=g\left(U_{x}, U_{y}, U_{z}\right)=g(U), \\
U_{x}=v_{x}-u_{x}(z), \quad U_{y}=v_{y}, \quad U_{z}=v_{z}, \\
g(U)=n\left[\frac{m \beta}{2 \pi}\right]^{3 / 2} \exp \left[-\beta m U^{2} / 2\right] .
\end{gathered}
$$

This PDF satisfies Eq. (27). When a mean velocity gradient exists, so that $u_{x}$ is such that its derivative with respect to $z$ does not vanish, Eq. (39) no longer complies with Eq. (27). Since the situation is time-independent, the ensuing (new) PDF cannot depend upon time, but will depend on $z$ (the direction of the velocity gradient). There are no external forces, so that $\dot{\mathbf{v}}$ vanishes. As a consequence, our Boltzmann equation (27) reduces to

$$
v_{z} \frac{\partial f}{\partial z}=-\tau^{-1}\left(f-f_{0}\right)
$$

One assumes that $\partial v_{x} / \partial z$ is small enough that $\partial f / \partial z$ is also small, so that

$$
f=f_{0}+f_{1}, \quad f_{1} \ll f_{0} .
$$

As a result, we find that

$$
f_{1}=-\tau v_{z} \frac{\partial f_{0}}{\partial z}
$$

It is clear from Eq. (39) that

$$
\frac{\partial f_{0}}{\partial z}=\frac{\partial g}{\partial U_{x}} \frac{\partial U_{x}}{\partial z}=-\frac{\partial g}{\partial U_{x}} \frac{\partial u_{x}}{\partial z},
$$

while

$$
\frac{\partial g}{\partial U_{x}}=-m \beta g U_{x}
$$

a relation that we will use below. Here, it will become clear that we need simply to write

$$
\frac{\partial f_{0}}{\partial z}=-\frac{\partial u_{x}}{\partial z} \frac{\partial g}{\partial U_{x}}
$$

so that

$$
f_{1}=\tau v_{z} \frac{\partial u_{x}}{\partial z} \frac{\partial g}{\partial U_{x}}=-m \beta \tau v_{z} U_{x} \frac{\partial u_{x}}{\partial z} f_{0},
$$

and, finally, 


$$
f=f_{0}+\tau v_{z} \frac{\partial u_{x}}{\partial z} \frac{\partial g}{\partial U_{x}}=f_{0}\left\{1-m \beta \tau v_{z} U_{x} \frac{\partial u_{x}}{\partial z}\right\} .
$$

Now, the $z x$ component of the stress is

$$
P_{z x}=m \int d^{3} v f U_{x} U_{z} .
$$

As $f_{0}$ depends only on the absolute value of $\mathbf{U}$, the above integral vanishes if one replaces $f$ by $f_{0}$ in the preceding integral for symmetry reasons. Thus,

$$
P_{z x}=m \frac{\partial u_{x}}{\partial z} \int d^{3} v v_{z} \tau \frac{\partial g}{\partial U_{x}} U_{x} U_{z} .
$$

According to Eq. (33), $v_{z}=U_{z}$, so that, assuming that the relaxation time does not depend upon velocity [30],

$$
\begin{aligned}
P_{z x} & =m \tau \frac{\partial u_{x}}{\partial z} \int d^{3} v \frac{\partial g}{\partial U_{x}} U_{x} U_{z}^{2} \\
& =m \tau \frac{\partial u_{x}}{\partial z} \iint d U_{y} d U_{z} U_{z}^{2} \int d U_{x} \frac{\partial g}{\partial U_{x}} U_{x} .
\end{aligned}
$$

Call the far right integral $A$. Using Eq. (44), we write it in the fashion

$$
A=-m \beta \int d U_{x} g U_{x}^{2} .
$$

As a consequence, using the equipartition theorem

$$
\begin{aligned}
P_{z x} & =-m^{2} \beta \tau \frac{\partial u_{x}}{\partial z} \int d^{3} U f_{0} U_{z}^{2} U_{x}^{2} \\
& =-m^{2} \beta \tau \frac{\partial u_{x}}{\partial z} n\left\langle U_{z}^{2}\right\rangle\left\langle U_{x}^{2}\right\rangle \\
& =-m^{2} \beta \tau \frac{\partial u_{x}}{\partial z} n(k T / m)^{2}=-\frac{\partial u_{x}}{\partial z} n \tau / \beta,
\end{aligned}
$$

and, for the coefficient of viscosity $\eta$, we finally get

$$
\eta=n \tau / \beta,
$$

in agreement with Eq. (38). Returning now to Eqs. (41) and (46), we stress that

$$
f=f_{0}\left[1-U_{z} U_{x} \tau m \beta \frac{\partial u_{x}}{\partial z}\right] .
$$

\section{The Rumer and Ryvkin treatment}

The Rumer and Ryvkin technique [27] is not the conventional one for dealing with the Boltzmann transport equation. However, it constitutes an essential ingredient in formulating our Fisher treatment of nonequilibrium problems. It is thus convenient, for illustrative purposes, to discuss the manner of using it within the context of our viscosity example.

To such an end, we start by remembering that the first two Hermite polynomials are

$$
H_{0}=1, \quad H_{1}=\frac{1}{\sqrt{2}} 2 x,
$$

and, with

$$
\phi(x, \omega)=\left[\frac{\omega}{\pi}\right]^{1 / 4} \exp \left[-x^{2} / 2\right]
$$

the first two members of the Gauss-Hermite basis (of $\mathcal{L}^{2}$ ) are

$$
\psi_{0}=H_{0} \phi, \quad \psi_{1}=H_{1} \phi .
$$

Since we have [30]

$$
n[m \beta /(2 \pi)]^{1 / 2} \exp \left[-\beta m v_{z}^{2} / 2\right]=f_{0, z}=n \psi_{0}^{2},
$$

our variables $x, \omega$ in Eqs. (55) and (56) are

$$
\omega=m \beta / 2, \quad 2 x=\sqrt{[2 \beta m]} v_{z},
$$

which allows us to recast Eq. (55) as

$$
H_{0}=1, \quad H_{1}=\sqrt{[\beta m]} v_{z} .
$$

We deal now with a three-dimensional problem. The pertinent Gauss-Hermite basis is the set of functions

$\left\{\psi_{0}\left(v_{x}\right) \psi_{0}\left(v_{y}\right) \psi_{0}\left(v_{z}\right)\left[1+\sum_{l, m, n} H_{l}\left(U_{x}\right) H_{m}\left(U_{y}\right) H_{n}\left(U_{z}\right)\right]\right\}$,

where $l, m, n$ run over all non-negative integers.

As data we have here

$$
P_{z x}=m \int d^{3} v f U_{z} U_{x}
$$

In the present instance, in view of Eq. (61), the RR recipe (22) to find $f$ [27] should be

$$
f(\mathbf{U})=f_{0}(\mathbf{U})\left[1+a H_{1}\left(U_{x}\right) H_{1}\left(U_{z}\right)\right]=f_{0}\left[1+a \beta m U_{x} U_{z}\right],
$$

with the coefficient $a$ to be determined from the here relevant velocity moment (61) and the prior knowledge expressed by Eq. (38). We thus evaluate Eq. (61) using the ansatz (62),

$$
P_{z x}=m \int d^{3} U\left\{f_{0}\left[1+a \beta m U_{x} U_{z}\right]\right\} U_{z} U_{x} .
$$

The integral $\int d^{3} U\left\{f_{0} U_{x} U_{z}\right]$ vanishes by symmetry. Thus,

$$
\begin{aligned}
P_{z x} & =a m^{2} \beta \int d^{3} U\left\{f_{0} U_{x}^{2} U_{z}^{2}\right\}=a m^{2} \beta n\left\langle U_{x}^{2}\right\rangle\left\langle U_{z}^{2}\right\rangle \\
& =\frac{a m^{2} n \beta}{[\beta m]^{2}} \\
& =n \frac{a}{\beta},
\end{aligned}
$$

where the equipartition theorem has been employed.

Since Eqs. (64) and (38) have to be equal, 


$$
a=-\tau \frac{\partial u_{x}}{\partial z}
$$

and

$$
f=f_{0}\left[1-U_{x} U_{z}\left(\tau \frac{\partial u_{x}}{\partial z} m \beta\right)\right]
$$

which is identical to Eq. (54).

\section{The SWE treatment}

\section{Ground state}

We start with the $z$ component of the probability amplitude that obeys

$$
\psi_{z}^{\prime \prime} / 2+\lambda_{1}(t)\left(v_{z}^{2} / 8\right) \psi_{z}=-(\alpha / 8) \psi_{z},
$$

and we set

$$
\lambda_{1}(t) / 8=\omega^{2} / 2 \quad \text { and } \quad(\alpha / 8)=-E
$$

so that the problem becomes time-independent. Our prior knowledge is the equipartition result

$$
\left\langle v_{z}^{2}\right\rangle=\frac{1}{\beta m}
$$

which entails, as discussed above, $\omega=\beta m / 2$, in view of the fact that the ground state of our SWE reads

$$
\psi_{0, z}=\left[\frac{\omega}{\pi}\right]^{1 / 4} \exp \left[-\omega v_{z}^{2} / 2\right] .
$$

Obviously, $\psi_{0, z}^{2}=f_{0, z}$, the $z$ component of the equilibrium PDF of the preceding subsections.

\section{Admixture of excited states}

We assume now that we have the additional piece of knowledge (38) for $P_{z x}$. Our SWE obeys now $\psi=\psi_{x} \psi_{y} \psi_{z}$ (and, also, $\psi_{0}=\psi_{0, x} \psi_{0, y} \psi_{0, z}$ ),

$$
\psi^{\prime \prime} / 2+\omega^{2}\left(v_{z}^{2} / 2\right) \psi+a U_{x} U_{z} \psi=E \psi,
$$

that can be treated perturbatively in view of our knowledge of the problem. $\quad a \ll 1$ is here the perturbation coupling constant.

It is well known [31] that, if one perturbs the ground state of the one-dimensional harmonic-oscillator wave function with a linear term, only the first excited state enters the perturbative series because of the selection rules [31],

$\left\langle\psi_{0} H_{n}(x)|x| \psi_{0} H_{m}(x)\right\rangle=c_{1} \delta(n, m+1)+c_{2} \delta(n, m-1)$,

where $c_{1}, c_{2}$ are appropriate constants [31], which entail that, for $n=0$ (ground state), only $m=1$ (first excited state) contributes [31]. As a consequence, we can write (up to first order in perturbation theory)

$$
\begin{aligned}
\psi & =\psi_{0}+\psi_{1}=\left[1+b H_{1}\left(U_{x}\right) H_{1}\left(U_{z}\right)\right] \psi_{0} \\
& =\left(1+b \beta m U_{x} U_{z}\right) \psi_{0},
\end{aligned}
$$

and, up to first-order terms as well,

$$
\psi^{2}=\left[1+2 b H_{1}\left(U_{x}\right) H_{1}\left(U_{z}\right)\right] \psi_{0}=\left[1+2 b(\beta m) U_{x} U_{z}\right] \psi_{0}
$$

We evaluate now $\left\langle\psi\left|U_{x} U_{z}\right| \psi\right\rangle$. For symmetry reasons it is obvious that $\left\langle\psi_{0}\left|U_{x} U_{z}\right| \psi_{0}\right\rangle=0$. Thus,

$$
\left\langle\psi\left|U_{x} U_{z}\right| \psi\right\rangle=2 \beta n m^{2}\left\langle\psi_{0}\left|U_{x}^{2} U_{z}^{2}\right| \psi_{0}\right\rangle
$$

Using now the equipartition result $\left\langle U_{x}^{2}\right\rangle_{0}\left\langle U_{z}^{2}\right\rangle_{0}=n /(m \beta)^{2}$, we arrive at

$$
P_{z x}=2 \beta b n m^{2}\left\langle\psi_{0}\left|U_{x} U_{z}\right| \psi_{0}\right\rangle=2 b n / \beta=a n / \beta, \quad a=2 b,
$$

which coincides with the RR result obtained in the preceding subsection. Thus,

$$
\psi^{2}=\left[1+a(\beta m) U_{x} U_{z}\right] \psi_{0}^{2}
$$

We have thereby recovered the RR result, which we previously verified to be correct in Sec. VIC.

\section{CONCLUSIONS AND DISCUSSION}

It is becoming increasingly evident $[4-7,11,14,15,25,26]$ that Fisher information $I$ is vital to the fundamental nature of physics. In a previous effort [2], we showed how the $I$ concept lays the foundation for thermodynamics in the usual equilibrium case. Here and in [26] we have shown that the nonequilibrium thermodynamics case can likewise be formed in this way. This considerably expands the horizon envisioned in [2].

The main result of this work is the establishment, by means of Fisher information, of a connection between nonequilibrium thermodynamics and quantum mechanics. The emphasis here lies in the word "connection." Why would such a link be of interest? Because it clearly shows that thermodynamics and quantum mechanics can both be expressed by a formal SWE (20), out of a common informational basis [21].

The physical meaning of this SWE is flexible, since its "potential function" $U(x)$ originates in data $\left\langle A_{k}\right\rangle_{t}$, via Eq. (21), of a physically general nature. This depends upon the application. The $\left\langle A_{k}\right\rangle_{t}$ are introduced into the theory as empirical inputs. The approach also encompasses quantum effects. In the latter cases, the effective potential function includes quantum effects. Also, the Planck constant $\hbar$, which does not explicitly appear in Eq. (20), would appear in one or more inputs $\left\langle A_{k}\right\rangle_{t}$ as, for example, would occur if the expectation value of the linear momentum of an electron were 
measured. The classical Boltzmann equation of the RR approach would then of course not be useable. In this way, our approach encompasses both quantum and classical thermodynamic effects.
Finally, and as a concrete example of the power of our abstract formalism, we have successfully applied it here to the nonequilibrium problem posed by the phenomenon of viscosity in dilute gases.
[1] E. T. Jaynes, in Statistical Physics, edited by W. K. Ford (Benjamin, New York, 1963); A. Katz, Statistical Mechanics (Freeman, San Francisco, 1967).

[2] R. Frieden, A. R. Plastino, A. Plastino, and B. H. Soffer, Phys. Rev. E 60, 48 (1999).

[3] R. A. Fisher, Proc. Cambridge Philos. Soc. 22, 700 (1925).

[4] B. R. Frieden, Am. J. Phys. 57, 1004 (1989).

[5] B. R. Frieden, Phys. Lett. A 169, 123 (1992).

[6] B. R. Frieden, in Advances in Imaging and Electron Physics, edited by P. W. Hawkes (Academic, New York, 1994), Vol. 90, pp. 123-204.

[7] B. R. Frieden, Physica A 198, 262 (1993).

[8] B. R. Frieden and R. J. Hughes, Phys. Rev. E 49, 2644 (1994).

[9] B. Nikolov and B. R. Frieden, Phys. Rev. E 49, 4815 (1994).

[10] B. R. Frieden, Phys. Rev. A 41, 4265 (1990).

[11] B. R. Frieden and B. H. Soffer, Phys. Rev. E 52, 2274 (1995).

[12] B. R. Frieden, Found. Phys. 21, 757 (1991).

[13] R. N. Silver, in E. T. Jaynes: Physics and Probability, edited by W. T. Grandy, Jr. and P. W. Milonni (Cambridge University Press, Cambridge, England, 1992).

[14] A. Plastino, A. R. Plastino, H. G. Miller, and F. C. Khana, Phys. Lett. A 221, 29 (1996).

[15] A. R. Plastino and A. Plastino, Phys. Rev. E 54, 4423 (1996).

[16] A. R. Plastino, A. Plastino, and H. G. Miller, Phys. Rev. E 56, 3927 (1997).
[17] A. Plastino, A. R. Plastino, and H. G. Miller, Phys. Lett. A 235, 129 (1997).

[18] B. R. Frieden and B. H. Soffer, Found. Phys. 13, 89 (2000).

[19] A. S. Holevo, Probabilistic and Statistical Aspects of Quantum Theory (North-Holland, Amsterdam, 1982), Chap. VI.

[20] M. Reginatto, Phys. Rev. A 58, 1775 (1998); Phys. Lett. A 249, 355 (1998).

[21] M. J. W. Hall, Phys. Rev. A 62, 012107 (2000).

[22] R. E. Nettleton, J. Phys. A 35, 295 (2002).

[23] C. Villani, J. Math. Pures Appl. 77, 821 (1998).

[24] M. Ravicule, M. Casas, and A. Plastino, Phys. Rev. A 55, 1695 (1997).

[25] A. R. Plastino, M. Casas, and A. Plastino, Phys. Lett. A 246, 498 (1998).

[26] R. Frieden, Physics from Fisher Information (Cambridge University Press, Cambridge, England, 1998).

[27] Y. B. Rumer and M. S. Ryvkin, Thermodynamics, Statistical Mechanics and Kinetics (MIR, Moscow, 1980).

[28] R. A. Fisher, Statistical Methods and Scientific Interference, 2nd ed. (Oliver and Boyd, London, 1959).

[29] P. I. Richards, Manual of Mathematical Physics (Pergamon Press, London, 1959), p. 342.

[30] F. Reif, Fundamentals of Statistical and Thermal Physics (McGraw-Hill, New York, 1965).

[31] J. M. Cassels, Basic Quantum Mechanics (McGraw-Hill, New York, 1970). 